\title{
Quality of life and tracheostomy influence in successfully treated hypopharyngeal cancer: A case report
}

\author{
ALINA-GEORGIANA VULCU CORDUNIANU ${ }^{1,2}$, GABRIEL GANEA ${ }^{2}$, \\ MIHAI ALEXANDRU CORDUNIANU ${ }^{1}$ and DANIEL COCHIOR ${ }^{1,3-5}$ \\ ${ }^{1}$ Medicine Doctoral School, Titu Maiorescu University, 040317 Bucharest; ${ }^{2}$ ENT Department, Carol Davila Central \\ University Emergency Military Hospital, 10825 Bucharest; ${ }^{3}$ Faculty of Medicine, Titu Maiorescu University \\ of Bucharest, 040441 Bucharest; ${ }^{4}$ General Surgery Department, Monza Clinical Hospital, 021967 Bucharest; \\ ${ }^{5}$ General Surgery Department, Sanador Clinical Hospital, 010991 Bucharest, Romania
}

Received September 3, 2021; Accepted October 5, 2021

DOI: $10.3892 /$ etm.2022.11118

\begin{abstract}
Squamous cell carcinoma (SCC) of the hypopharynx is associated with the worst prognosis of all squamous cell cancer types of the head and neck, with a rich submucosal lymphatic network and a significant inferior extent. Generally, cancers of the head and neck have a big impact on the quality of life $(\mathrm{QoL})$ in patients, both before and after treatment, due to the fact that most cases are diagnosed in advanced stages, with lymph node metastases and have an aggressive pattern associated with low survival rates. The present case report aimed to see the evolution of a patient successfully treated for hypopharyngeal cancer with conservation therapies from the QoL perspective and the influence made by the presence of tracheostomy. Variables, including clinical parameters (sex, age, TNM classification, performance status, histological type and tumor subsite), local control, QoL, Adult Comorbidity Evaluation-27 score and overall survival were assessed, according to the American Joint Committee on Cancer 8th edition, for a 58-year-old patient treated with conservation therapies and tracheostomy in a tertiary center. Subjective features measured through the QoL questionnaire had scores with lowering tendencies during the treatment period. The patient was provided with early training for tracheostomy care and early discharge education. The following objective features of treatment response were exhibited: Hemoglobin levels, leukocyte count, tumor size, imagistic evaluations and local control to confirm treatment response. It is essential when planning the treatment of aggressive and rare pathologies, such as hypopharyngeal cancer, to consider the QoL and patient choice for better adherence. Overall, the results demonstrated
\end{abstract}

Correspondence to: Dr Alina-Georgiana Vulcu Cordunianu, Medicine Doctoral School, Titu Maiorescu University, 16 Gheorghe Sincai Boulevard, 040317 Bucharest, Romania

E-mail: alina.georgiana.vulcu@gmail.com

Key words: hypopharyngeal cancer, organ conservation therapy, chemoradiotherapy, quality of life, tracheostomy that the initial requirement for emergency tracheostomy was not associated with any ulterior complications and did not influence the end result of the treatment.

\section{Introduction}

Hypopharyngeal cancer is a rare, aggressive pathology usually detected in advanced stages due to the absence of specific symptoms. Because of this late diagnosis, the treatment modalities are usually reduced (1). One of the most important decision-making factors involving treatment options for patients is the associated survival rate. However, there are pathologies, including hypopharyngeal cancer in which the treatment modalities (primary surgery, partial or total pharyngectomy with laryngectomy followed by radiation therapy; or non-surgical, chemoradiation therapy; initial radiation therapy followed by surgery) have similar survival probabilities and in situations like this, quality of life (QoL) becomes an essential criterion in treatment selection $(2,3)$.

In Europe, this malignancy has an incidence rate of $1.3 / 100.000$, with a 5-year relative survival rate of only $25 \%$ (4). Hypopharyngeal cancer is associated with a high rate of mortality (up to $30 \%$ in the first year of diagnosis) and a low rate of survival to 5 years ( $<40 \%$ of all patients) (1). Factors closely associated with this pathology are tobacco use and alcohol consumption (4); however, other factors, such as genetic predisposition, diet, human papilloma virus infection, Plummer-Vinson or Paterson-Brown-Kelly syndrome are also associated with the development of this disease (1). Furthermore, additional environmental and clinical risk factors, including asbestos (5), formaldehyde and coal dust (6), cannot be ruled out, and the clinician must take into consideration that the reverse situation, where genetic predisposition is augmented by environmental factors, is even more common and complicated (7).

Due to the lack of early symptoms in this region, up to $75 \%$ of newly diagnosed patients are in stage III and IV (8). Another important aspect is that upstaging to stage T4b is usually done during the surgery when the involvement of the prevertebral fascia is revealed (1). Some of the most common symptoms in advanced stages are presence of neck mass, 
airway distress, dysphagia, gastroesophageal reflux and sore throat or referred otalgia (1). Non-surgical treatment options include primary radiotherapy (RT), chemo-radiation (CRT) and surgical options, laser surgery (early stages) or a total laryngectomy with partial or total pharyngectomy (9).

\section{Case report}

The present case report followed the evolution of a 58-year-old male patient, with a history of smoking and alcohol consumption, that was diagnosed and treated for squamous cell carcinoma (SCC) of the hypopharynx between September 2019 and March 2021 at the Carol Davila Central University Emergency Military Hospital, Bucharest. The patient was admitted to the emergency unit for airway distress, dysphonia and dysphagia and was moved to the ear, nose and throat (ENT) clinic for investigations and treatment. Emergency tracheostomy and biopsy of the lesion were performed, along with computed tomography of the head and neck. ENT assessment revealed a hypopharyngeal tumor located in the right piriform sinus and lateral pharyngeal wall, which spread to the right vocal cord with a mass effect and reduced respiratory flow (Fig. 1). Computed tomography revealed pharyngeal wall thickening, with a reduced pharyngeal lumen and a nonhomogeneous anterior laryngeal mass of 2.2-2.4 cm, with uneven margins, between the median part of the thyroid cartilages with a lithic effect on them. The histopathology report diagnosed the mass as SCC, well differentiated and invasive.

Patient evaluations included laboratory testing for the following: Complete blood count; coagulation profile (INR, TQ and AP); erythrocyte sedimentation rate (ESR), glucose, urea, creatinine levels; bilirubin; serum alanine aminotransferase (ALT); serum aspartate aminotransferase (AST), sodium, potassium, serum amylase and C-reactive protein (CRP). Questionnaires were used to assess patient outcome, including Adult Comorbidity Evaluation-27 (ACE-27) for significant ailments (10), Karnofsky Performance Status Scale (KPSS) (11) for functional impairment and better understand patient requirements and the European Organization for Research and Treatment of Cancer Quality of Life Questionnaire (12) with the Head and Neck Cancer Module (EORTC QLQ-H\&N35), with the commission's approval for use.

Treatment options were based on parameters, such as age, comorbidities, the personal absence history of cancer, objective tumor parameters [histology, location, TNM classification Stage IV A (T4aNOM0) (13), ACE27-2/moderate], clinical and imaging aspects, and also subjective parameters, including personal choice, expected result and QoL (4). Optimal treatment strategies were discussed by a multidisciplinary team, who considered all aforementioned factors. The treatment regimen used was concurrent (C)CRT, using the volumetric modulated arc therapy (VMAT), as radiation technique in a total dose of 70.2 Gy in 39 fractions, five fractions per week $(1.8 \mathrm{~Gy} / \mathrm{fr})$ and six series of chemotherapy using cisplatin ( $70 \mathrm{mg} / \mathrm{session})$. The patient did not require dose alteration for sensory neuropathy, neutropenia or grade 3 toxicity. The patient was assessed for treatment response via clinical/endoscopic examination and computed tomography/magnetic resonance imaging during radiation and chemotherapy at 3,6 and 12 months following completion of treatment. Following treatment, the patient was in complete remission able to speak and breathe without the tracheal cuff.

Tracheostomy modifies the QoL through the inability to speak, difficulties swallowing and body image alterations (14). To prevent further complications, such as bleeding, accidental decannulation and occlusion, it is essential that the patient benefits from discharge training and help from a family member (15). In the present study, the patient and his wife were trained during the hospital stay for tracheostomy care and discharge education. No complaints or complications involving the tracheostomy were observed. The clinical trials that support the use of induction chemotherapy for hypopharyngeal cancer are not consistent as they have failed to exhibit a significant improvement and as a result, CCRT is used as a therapeutic option for a greater local and regional control $(16,17)$.

Follow-up surveillance after the completion of chemoradiation was at 3, 6, 9, 12 and 18 months, showing remission with no distant metastasis evaluated through the imaging reports (magnetic resonance imaging and computed tomography). The future plan for the patient is closing the tracheostomy, 2 months after cuff removal.

Hemoglobin levels varied throughout the study, from $12.7 \mathrm{~g} / \mathrm{dl}$ in the initial visit, which decreased during CCRT (11.8-10.2 $\mathrm{g} / \mathrm{dl})$ and increased after treatment completion (11.2 g/dl after 8 months and $13 \mathrm{~g} / \mathrm{dl}$ after 14 months). Leucocyte count decreased during CCRT; however, no significant alterations were observed before or after this interval. Platelet number was in between limits during the study, and inconclusive as an independent factor. Other variables, such as INR, TQ, AP, ESR, glucose, urea, creatinine levels, bilirubin, serum alanine aminotransferase, serum aspartate aminotransferase, sodium, potassium, serum amylase and C-reactive protein had variations within limit standards in all evaluations. A common side effect of radiation therapy is the sensory impairment of the mucosa, which is an important etiological mechanism in swallowing disorders and aspiration $(1,18)$. In addition, both surgical treatment and radiation therapy can alter laryngeal innervation and functions (10) (Table I).

The patient completed the Romanian version of the EORTC QLQ-H\&N35 questionnaire at each visit in the ENT clinic, which consists of 35 items allocated in 11 single item scales (teeth, opening mouth and dry mouth) and seven multi-item scales, measuring symptoms, such as pain, swallowing, taste and smell and speech. The questionnaire is reliable and valid, according to previous studies $(19,20)$. The scores of the questionnaire range from $0-100$. In the symptom scales, higher values correspond to a lower QoL and a greater issue (12). The items with the highest consistent raw scores were swallowing, speech and social contact and discomfort, with values that decreased with time. Social contact was partially modified by the global pandemic and legal restrictions. In addition, the patient chose not to answer the sexuality section.

ACE-27 is used to evaluate future development of severe acute toxicities in patients with head and neck cancer treated with chemoradiation therapy (10). This tool can also be used in patients who receive primary surgical treatment for SCC, as a prognostic indicator of mortality and complications (21). Higher grading of pre-existent pathologies is associated with higher chances of severe acute toxicities, postoperative surgical 
Table I. Raw score variations for EORTC QLQ-H\&N35.

\begin{tabular}{|c|c|c|c|c|}
\hline Symptom/item & $\begin{array}{l}\text { Raw score at } \\
\text { initial visit }\end{array}$ & $\begin{array}{l}\text { Raw score } \\
\text { before CRT }\end{array}$ & $\begin{array}{l}\text { Raw score } \\
\text { after CRT }\end{array}$ & $\begin{array}{c}\text { Raw score } 1 \text { year } \\
\text { after CRT }\end{array}$ \\
\hline Pain & 2.00 & 1.75 & 1.00 & 1.00 \\
\hline Swallowing & 3.00 & 2.00 & 1.25 & 1.00 \\
\hline Teeth & 1.00 & 1.00 & 1.00 & 1.00 \\
\hline Opening mouth & 1.00 & 1.00 & 1.00 & 1.00 \\
\hline Dry mouth & 1.00 & 1.00 & 2.00 & 1.00 \\
\hline Sticky saliva & 1.00 & 1.00 & 2.00 & 1.00 \\
\hline Senses & 1.00 & 1.00 & 1.5 & 1.00 \\
\hline Coughing & 3.00 & 3.00 & 2.00 & 1.00 \\
\hline Felt ill & 4.00 & 2.00 & 1.00 & 1.00 \\
\hline Speech & 4.00 & 4.00 & 2.33 & 1.66 \\
\hline Social eating & 3.25 & 3.00 & 1.25 & 1.00 \\
\hline Social contact & 3.00 & 3.40 & 2.60 & 2.60 \\
\hline Sexuality & - & - & - & - \\
\hline Pain killers & 2.00 & 2.00 & 1.00 & 1.00 \\
\hline Nutritional supplements & 1.00 & 1.00 & 1.00 & 1.00 \\
\hline Feeding tube & 1.00 & 1.00 & 1.00 & 1.00 \\
\hline Weight loss & 2.00 & 2.00 & 1.00 & 1.00 \\
\hline Weight gain & 1.00 & 1.00 & 2.00 & 2.00 \\
\hline
\end{tabular}

CRT, chemoradiotherapy; -, not provided.
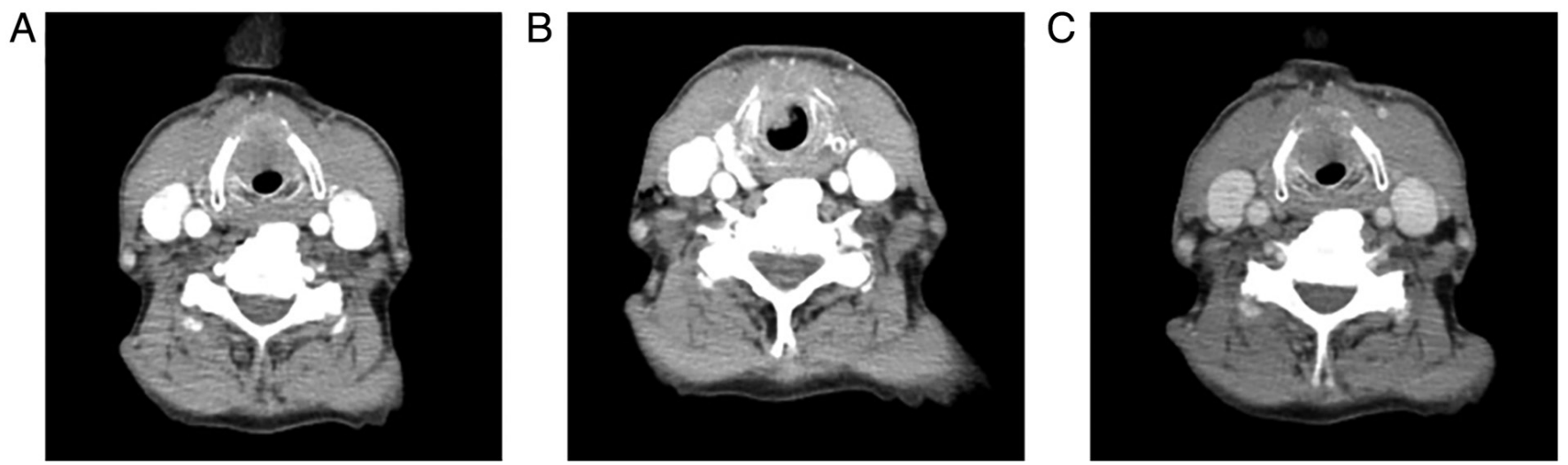

Figure 1. Initial tomographic report. (A) Nonhomogeneous mass with lithic effect on the median part of the thyroid cartilage. (B) The mass reduced the laryngeal lumen and airflow. (C) The lithic effect is present on all the median part of the thyroid cartilage.
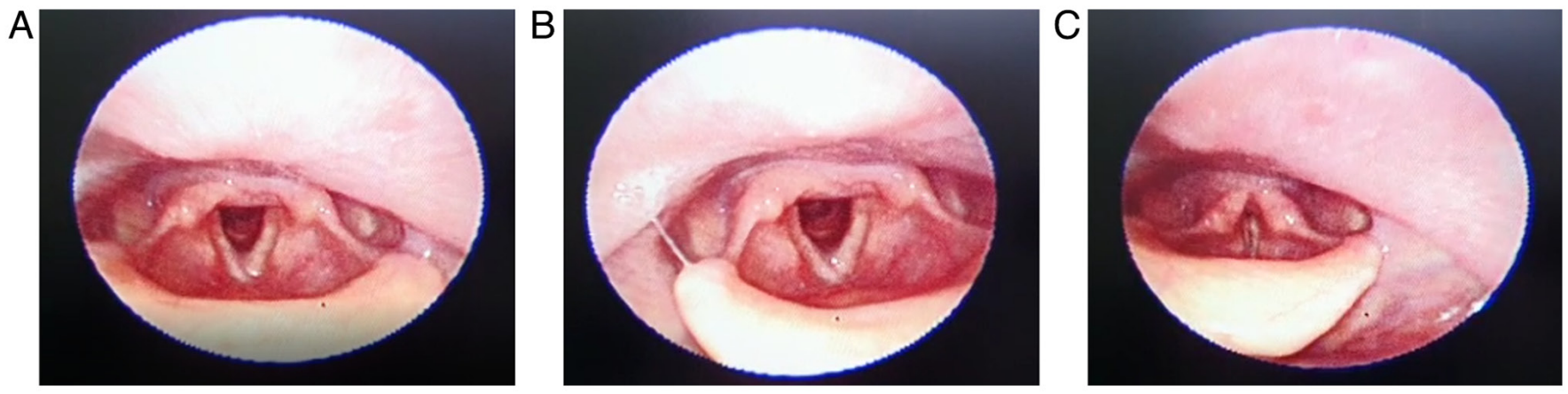

Figure 2. Local aspect 1 year after chemoradiation therapy. (A and B) Endoscopic view of hypopharynx and larynx in abduction (during breathing). (C) Endoscopic view in adduction (during speaking). 
complications and mortality rate $(17,18)$. In the present study, ACE-27 indicated a moderate decompensation (grade 2) for cardiovascular system. KPSS values varied from $20 \%$ in the initial visit (the patient required active treatment) to $70 \%$ in the last visit (he managed to care for himself although he could not actively work anymore) (Fig. 2).

\section{Discussion}

Given the assumption that both treatment and technique help performance, and medical research can sometimes require a high degree of abstraction (22); studies so far were not able to show a significant improvement of the survival rates and local control using various regimens of induction chemotherapy in comparison to the concurrent CRT, the last one remains the standard of care in organ conservation for advanced hypopharyngeal cancer (16).

Other studies have already proven that ACE-27 score, BMI, hemoglobin and albumin levels are independent variables of prognosis in cancer and are useful in estimating survival and distinguishing between risk groups $(9,21,23,24)$. In the present study, a good response in QoL was associated with values that had lowering tendencies throughout evaluations. The highest scores that accounted the most discomfort were in swallowing, speech and social contact, similar to other QoL studies $(14,19,20)$. The requirement for emergency tracheostomy did not predict future complications; early tracheostomy care and discharge education had an important role in maintaining good QoL. In addition, good treatment response was associated with normal hemoglobin levels at the end of treatment and within standard limits for platelet number, coagulation profile, urea, creatinine levels, ALT, AST, sodium, potassium, CRP and ESR, for the entire evaluation period.

Further studies are required to validate the results presented here. Prospective studies will apply this template on a larger sample size from the QoL perspective and treatment response.

\section{Acknowledgements}

This material is part of a larger, on-going doctoral study by A.G. Vulcu Cordunianu, a PhD student at Titu Maiorescu University of Bucharest, Romania, Medicine Doctoral School with Cochior Daniel University Professor, PhD Habil, as thesis coordinator.

\section{Funding}

No funding was received.

\section{Availability of data and materials}

The datasets used and analyzed during the current case are available from the corresponding author on reasonable request.

\section{Authors' contributions}

AGVC and DC drafted the presentation and were involved in the design and conception of the present study. GG and AGVC obtained patient consent, acquired the data and confirmed authenticity of all the raw data. MAC was responsible for data analysis and performed the literature review. AGVC and MAC drafted the initial manuscript. DC critically revised the manuscript for important intellectual content. All authors have read and approved the final manuscript.

\section{Ethics approval and consent to participate}

Not applicable.

\section{Patient consent for publication}

The patient provided written informed consent for his data collection and submission for publication.

\section{Competing interests}

The authors declare that they have no competing interests.

\section{References}

1. Vila PM and Uppaluri R: Neoplasms of the hypopharynx and cervical esophagus. In: Cummings Otolaryngology Head and Neck Surgery. Flint PW and Cummings CW (eds). 6th edition, Vol II. pp1537-1553, Elsevier Saunders, Philadelphia, 2015.

2. Firkins J, Hansen L, Driessnack M and Dieckmann N: Quality of life in 'cronic' cancer survivors: A meta-analysis. J Cancer Survivorship 14: 504-517, 2020.

3. Lewandowska A, Rudzki G, Lewandowski T, Prochnicki M, Rudzki S, Laskowska B and Brudniak J: Quality of life of cancer patients treated with chemotherapy. Int J Environ Res Public Health 17: 6938, 2020.

4. Machiels JP, René Leemans C, Golusinski W, Grau C, Licitra L, Gregoire V; EHNS Executive Board. Electronic address: secretariat@ehns.org; ESMO Guidelines Committee. Electronic address: clinicalguidelines@esmo.org and ESTRO Executive Board. Electronic address: info@estro.org: Squamous cell carcinoma of the oral cavity, larynx, oropharynx and hypopharynx: EHNS-ESMO-ESTRO Clinical Practice Guidelines for diagnosis, treatment and follow-up. Ann Oncol 31: $1462-1475,2020$

5. Marchlang JL, Luce D, Leclerc A, Goldberg P, Orlowski E, Bugel I and Brugère J: Laryngeal and hypopharyngeal cancer and occupational exposure to asbestos and man-made vitreous fibers: Results of a case-control study. Am J Ind Med 37: 581-589, 2000.

6. Laforest L, Luce D, Goldberg P, Begin D, Gerin M, Demers PA, Brugere $\mathrm{K}$ and Leclerc A: Laryngeal and hypopharyngeal cancers and occupational exposure to formaldehyde and various dusts: A case-control study in France. Occup Environ Med 57: 767-773, 2000

7. Neagu A, Mocanu A, Bonciu A, Coada G and Mocanu H: Prevalence of GJB2 gene mutations correlated to presence of clinical and environmental risk factors in the etiology of congenital sensorineural hearing loss of the Romanian population. Exp Ther Med 21: 612, 2021.

8. Petersen JF, Timmermans AJ, van Dijk BAC, Overbeek LIH, Smit LA, Hilgers FJM, Stuiver MM and van den Brekel MWM: Trends in treatment, incidence and survival of hypopharynx cancer: A 20-year population-based study in the Netherlands. Eur Arch Otorhinolaryngol 275: 181-189, 2018.

9. Arends CR, Petersen JF, van der Noort V, Timmermans AJ, Leemans CR, de Bree R, Van den Brekel MWM and Stuiver MM: Survival predictions of hypopharynx cancer: Development of a clinical prediction model. Laryngoscope 130: 2166-2172, 2020.

10. Monteiro AR, Garcia AR, Pereira TC, Macedo F, Soares RF, Pereira K, Serra T, Khouri L, Ribeiro J and Teixeira MM: ACE-27 as a prognostic tool of severe acute toxicities in patients with head and neck cancer treated with chemoradiotherapy: A real-world, prospective, observational study. Support Care Cancer 29: 1863-1871, 2021. 
11. Friedlander AH and Ettinger RL: Karnofsky performance status scale. Spec Care Dentist 29: 147-148, 2009.

12. Singer S, Arraras JI, Chie WC, Fisher SE, Galalae R, HammerlidE, Nicolatou-Galitis O, Schmalz C, Verdonck-de Leeuw I, Gamper E, et al: Performance of the EORTC questionnaire for the assessment of quality of life in head and neck cancer patients EORTC QLQ-H\&N35: A methodological review. Qual Life Res 22: 1927-1941, 2013.

13. American Joint Committee on Cancer: Oropharynx and hypopharynx. In: AJCC Cancer Staging Manual. 8th edition. Springer, New York, NY, p16123-16135, 2017.

14. Seyman CC and Celik SS: Effect of protective cover on the quality of life of patients with tracheostomy-A quasi-experimental study. J Perianesth Nurs 35: 430-436, 2020.

15. Colandrea $\mathrm{M}$ and Eckardt P: Improving tracheostomy care delivery: Instituting clinical care pathways and nursing education to improve patient outcomes. ORL Head Neck Nurs 34: 7-16, 2016.

16. Lim SH, Sun JM, Hong J, Oh D, Ahn YC, Chung MK, Jeong HS, Son YI, Ahn MJ, Baek CH, et al: Induction chemotherapy followed by concurrent chemoradiotherapy versus CCRT for locally advanced hypopharynx and base of tongue cancer. Korean J Intern Med 36 (Suppl 1): S217-S224, 2021.

17. Yokota T, Shibata M, Hamauchi S, Shirasu H, Onozawa Y, Ogawa $\mathrm{H}$, Onoe $\mathrm{T}$, Kawakami $\mathrm{T}$, Turuta $\mathrm{M}$, Inoue $\mathrm{H}$, et al: Feasibility and efficacy of chemoradiotherapy with concurrent split-dose cisplatin after induction chemotherapy with docetaxel/cisplatin/5-fluorouracil for locally advanced head and neck cancer. Mol Clin Oncol 13: 35, 2020.
18. Pezdirec M, Strojan P and Boltezar IH: Swallowing disorders after treatment for head and neck cancer. Radiol Oncol 53: 225-230, 2019.

19. Sherman AC, Simonton S, Adams DC, Vural E, Owens B and Hanna E: Assessing quality of life in patients with head and neck cancer: cross-validation of the European Organization for Research and Treatment of Cancer (EORTC) Quality of Life Head and Neck module (QLQ-H\&N35). Arch Otolaryngol Head Neck Surg 126: 459-467, 2000

20. Gomes EPAA, Aranha AMF, Borges AH and Volpato LER Head and Neck cancer Patients' quality of life: Analysis of three instruments. J Dent (Shiraz) 21: 31-41, 2020.

21. Milne S, Parmar J and Ong TK: Adult comorbidity Evaluation-27 as a predictor of postoperative complications, two-year mortality, duration of hospital stay, and readmission within 30 days in patients with squamous cell carcinoma of the head and neck. $\mathrm{Br}$ J Oral Maxillofac Surg 57: 214-218, 2019.

22. Mocanu H, Mocanu AI, Drăgoi AM and Rădulescu M: Long-term histological results of ossicular chain reconstruction using bioceramic implants. Exp Ther Med 21: 260, 2021.

23. Caro JJ, Salas M and Goss G: Anemia as independent prognostic factor for survival in patients with cancer: A systemic, quantitative review. Cancer 91: 2214-2221, 2001.

24. Danan D, Shonka DC Jr, Selman Y, Chow Z, Smolkin ME and Jameson MJ: Prognostic value of albumin in patients with head and neck cancer. Laryngoscope 126: 1567-1571, 2016.

This work is licensed under a Creative Commons Attribution-NonCommercial-NoDerivatives 4.0 International (CC BY-NC-ND 4.0) License. 